\title{
Restriction Spectrum Imaging Improves Risk Stratification in Patients with Glioblastoma
}

\author{
D.P. Krishnan, (D) R. Karunamuni, (D).M. Leyden, (D).M. Seibert, (D) R.L. Delfanti, (D).M. Kuperman, (D) H. Bartsch, (DP. Elbe, \\ (D) A. Srikant, A.M. Dale, (D). Kesari, (DD.E. Piccioni, (D).A. Hattangadi-Gluth, (DN. Farid, DC.R. McDonald, and (D).S. White
}

\begin{abstract}
BACKGROUND AND PURPOSE: ADC as a marker of tumor cellularity has been promising for evaluating the response to therapy in patients with glioblastoma but does not successfully stratify patients according to outcomes, especially in the upfront setting. Here we investigate whether restriction spectrum imaging, an advanced diffusion imaging model, performed after an operation but before radiation therapy, could improve risk stratification in patients with newly diagnosed glioblastoma relative to ADC.
\end{abstract}

MATERIALS AND METHODS: Pre-radiation therapy diffusion-weighted and structural imaging of 40 patients with glioblastoma were examined retrospectively. Restriction spectrum imaging and ADC-based hypercellularity volume fraction (restriction spectrum imagingFLAIR volume fraction, restriction spectrum imaging-contrast-enhanced volume fraction, ADC-FLAIR volume fraction, ADC-contrastenhanced volume fraction) and intensities (restriction spectrum imaging —FLAIR 90th percentile, restriction spectrum imaging-contrastenhanced 90th percentile, ADC-FLAIR 10th percentile, ADC-contrast-enhanced 10th percentile) within the contrast-enhanced and FLAIR hyperintensity VOIs were calculated. The association of diffusion imaging metrics, contrast-enhanced volume, and FLAIR hyperintensity volume with progression-free survival and overall survival was evaluated by using Cox proportional hazards models.

RESULTS: Among the diffusion metrics, restriction spectrum imaging-FLAIR volume fraction was the strongest prognostic metric of progression-free survival $(P=.036)$ and overall survival $(P=.007)$ in a multivariate Cox proportional hazards analysis, with higher values indicating earlier progression and shorter survival. Restriction spectrum imaging-FLAIR 90th percentile was also associated with overall survival $(P=.043)$, with higher intensities, indicating shorter survival. None of the ADC metrics were associated with progression-free survival/overall survival. Contrast-enhanced volume exhibited a trend toward significance for overall survival $(P=.063)$.

CONCLUSIONS: Restriction spectrum imaging-derived cellularity in FLAIR hyperintensity regions may be a more robust prognostic marker than ADC and conventional imaging for early progression and poorer survival in patients with glioblastoma. However, future studies with larger samples are needed to explore its predictive ability.

ABBREVIATIONS: $\mathrm{CE}=$ contrast-enhanced; $\mathrm{CPH}=$ Cox proportional hazards; $\mathrm{FLAIR}-\mathrm{HI}=$ FLAIR hyperintensity; $\mathrm{GBM}=$ glioblastoma; $\mathrm{HC}=$ hypercellularity; $\mathrm{HR}=$ hazard ratio; $\mathrm{MRSI}=\mathrm{MR}$ spectroscopic imaging; $\mathrm{PFS}=$ progression-free survival; $\mathrm{OS}=$ overall survival; $\mathrm{RSI}=$ restriction spectrum imaging; $\mathrm{RT}=$ radiation therapy; $\mathrm{vf}=$ volume fraction; vol = volume

lioblastoma (GBM) is the most common and aggressive malignant primary brain tumor. Unfortunately, there has been

Received June 21, 2016; accepted after revision December 9.

From the Multimodal Imaging Laboratory (A.P.K., K.M.L., T.M.S., J.M.K., H.B., P.E., A.S., A.M.D., N.F., C.R.M., N.S.W.) and Departments of Radiation Medicine (R.K., T.M.S.,

J.A.H.-G., C.R.M.), Radiology (R.L.D., J.M.K., H.B., A.M.D., N.F., N.S.W.), Neurosciences (A.M.D., D.E.P.), and Psychiatry (C.R.M.), University of California, San Diego, La Jolla, California; and Department of Translational Neuro-Oncology and Neurotherapeutics (S.K.), John Wayne Cancer Institute and Pacific Neuroscience Institute at Providence Saint John's Health Center, Santa Monica, California.

C.R. McDonald and N.S. White contributed equally to this work as co-senior authors.

This work was supported by the National Science Foundation grant 1430082 (N.S.W.); GE Healthcare, Investigator Initiated Research Award BOK92325 (N.S.W.); National Institutes of Health grants R01NS065838 (C.R.M.), RC2 DA29475 (A.M.D.), and EB00790-06 (A.M.D.); National Cancer Institute, Cancer Center Specialized Grant P30CA023100 (C.R.M.); National Institutes of Health ULITR000100 (J.A.H.-G.) only incremental improvement in the 5-year survival rate in the past decade. ${ }^{1}$ The standard of care for newly diagnosed GBM remains fairly uniform, with maximal permissible surgical resection followed by radiation therapy (RT) with concurrent and adjuvant temozolomide. ${ }^{2}$ Currently, novel molecular and cellular targeted therapies for treating GBMs are being investigated with many of

and KL2TR00144 (J.A.H.-G.); American Cancer Society Award ACS-IRG 70-002 (J.A.H.-G.); and American Cancer Society Research Scholar Grant RSG-15-229-01CCE (C.R.M.)

Please address correspondence to N.S. White, PhD, Multimodal Imaging Laboratory, Center for Multimodal Imaging and Genetics (CMIG), Floor 4W, 9452 Medical Center Dr, La Jolla, CA 92037; e-mail: nswhite@ucsd.edu

- Indicates open access to non-subscribers at www.ajnr.org

Indicates article with supplemental on-line photo.

http://dx.doi.org/10.3174/ajnr.A5099 
them now in phase II clinical trials. ${ }^{3}$ With the advent of these new therapies and a recent study showing that radiation dose escalation to $75 \mathrm{~Gy}$ (above the standard dose of $60 \mathrm{~Gy}$ ) is safe and possibly more effective in newly diagnosed GBM, ${ }^{4}$ stratification of patients at the highest risk for early progression is imperative because more aggressive or experimental treatments may be pursued in these individuals. These treatment decisions are usually considered within the first several weeks postsurgery once any residual tumor has been identified, making the pre-RT imaging pivotal for guiding the course of treatment.

Conventional MR imaging, including T1-postcontrast and FLAIR, is nonspecific because the former represents the breakdown of the blood-brain barrier due to tumor- and non-tumorrelated causes, and the latter may represent tumor-related edema, postradiation change, or any cause of gliosis. Advanced MR imaging techniques, such as DWI, may offer more specific information related to the underlying physiology of the tissue and may complement existing measures. ADC estimates the magnitude of water diffusion in relation to the physical barriers in its environment. It is frequently used as an imaging biomarker for tumor cellularity ${ }^{5,6}$ and is inversely correlated with tumor cell density. ${ }^{7}$ However, at the typical b-values used clinically $\left(b=0,1000 \mathrm{~s} / \mathrm{mm}^{2}\right)$, the diffusion signal primarily arises from the extracellular space. ${ }^{8}$ Therefore, in addition to estimating cell density, the $\mathrm{ADC}$ calculated at these b-values is also influenced by factors such as edema and necrosis, subsequently making ADC a rather nonspecific measure of tumor cellularity.

To account for the influence of edema and necrosis on ADC intensities in the tumor and peritumoral regions, histogram analysis of normalized ADC intensities ${ }^{9,10}$ (normalized with respect to mean $\mathrm{ADC}$ in normal-appearing white matter) and 2 Gaussian mixture modeling of the ADC intensities within the tumor ${ }^{5,6}$ have been proposed. However, these statistical methods seek only to reclassify voxels within an ROI so that voxels with presumably solid tumor are included in the analysis, while potentially problematic voxels that are confounded by partial voluming with edema and necrosis are removed. These methods have shown some promise for evaluating treatment response and predicting progression-free survival (PFS) in both the upfront ${ }^{5,11}$ and recurrent $^{6,12}$ setting following treatment with antiangiogenic therapy. However, the utility of these ADC metrics for predicting response to standard chemoradiation has been less frequently explored. Some data suggest that though ADC intensities are not predictive of PFS or overall survival (OS) in the upfront setting, ${ }^{5,10}$ the volume of ADC with a large tumor burden (normalized $\mathrm{ADC}<1.5$; hypercellularity $[\mathrm{HC}]$ volume) within the T2 volume stratifies OS both pre- ${ }^{13}$ and postsurgery. ${ }^{9}$ However, it is unclear whether the hypercellularity volume was correlated with the underlying T2 volume and whether its predictive value merely reflects the association of the T2 volume with survival. Multiple studies have used the increase or decrease of HC volume fraction (ie, the HC volume defined with respect to the variation in a mixture of normalappearing white and gray matter) as a predictive marker for evaluating treatment response ${ }^{14}$ because this metric may capture the percentage of the tumor that is highly cellular and correlated with the structural volumes. However, the utility of the HC volume fraction at individual time points for early risk stratification has not been explored, to our knowledge.
Table 1: Patient characteristics

\begin{tabular}{ll}
\hline & \multicolumn{1}{c}{$\begin{array}{c}\text { Resection Type: STR }(\boldsymbol{n}=22) ; \\
\text { GTR }(\boldsymbol{n}=18)^{\mathrm{a}}\end{array}$} \\
\hline Sex & 24 Male; 16 female \\
Age (median) (range) (yr) & $58(31-84)$ \\
PFS (median) (range) (mo) & $8.42(3.5-50.53) ; 7$ censored \\
OS (median) (range) (mo) & $19.48(6.37-50.93) ; 8$ censored \\
Bevacizumab at recurrence & 17 Patients \\
MGMT status & 12 Unmethylated, 8 methylated, \\
& 20 unknown \\
IDH status & 15 WT, 25 unknown \\
EGFR amplification & 7 Unamplified, 12 amplified, \\
& 21 unknown \\
EGFRVIII status & 12 Positive, 9 negative, 19 unknown \\
\hline
\end{tabular}

Note:-STR indicates subtotal resection; GTR, gross total resection; WT, wild type. a Resection type was determined from the immediate postsurgical scan acquired within 48 hours of the operation for all patients, which included both T1-postcontrast and FLAIR sequences.

Multicompartment models of diffusion based on advanced multishell acquisitions can provide a more straightforward approach for mitigating the confounding effects of edema and necrosis at the voxel level. In particular, restriction spectrum imaging (RSI) is an advanced diffusion imaging model that separates the relative contributions of hindered and restricted signals originating from extracellular and intracellular water compartments, respectively, by using a multi-b-shell acquisition in conjunction with a linear mixture model. ${ }^{15-17}$ Furthermore, RSI incorporates geometric information to disambiguate isotropic-restricted diffusion in tumor cells from anisotropic-restricted diffusion in elongated neuronal processes (axons/dendrites collectively called "neurites"). Previous studies have demonstrated the increased sensitivity and specificity of RSI over ADC and DWI in both brain tumors ${ }^{18}$ and prostate cancer, ${ }^{19}$ and McDonald et al ${ }^{20}$ have recently demonstrated that RSI cellularity is a stronger predictor of both PFS and OS in patients following treatment with bevacizumab relative to ADC. However, its utility for predicting survival in patients newly diagnosed with GBM has not been explored, to our knowledge.

Here we investigate the application of RSI for risk stratification in newly diagnosed, resected GBMs. Our hypothesis was that RSI, due to its multi-b-shell acquisition and its inherent ability to decouple diffusion signal within tumor cells from that of extracellular pathology (eg, edema), would be a more robust marker of patient outcomes.

\section{MATERIALS AND METHODS}

This institutional review board-approved retrospective study included 45 patients with pathologically confirmed primary GBM who had pre-RT MRIs (median, 23 days; range, 9-113 days from the operation; median, 10 days; range, 1-29 days before start of RT) that included standardized RSI and conventional imaging sequences acquired between January 2011 and November 2015. All patients were followed for at least 6 months (May 2016). Patient characteristics are shown in Table 1. PFS and OS were defined relative to the pre-RT scan. All scans were reviewed by a neuroradiologist to ensure image quality and determine the basis for exclusion. Of the 45 eligible candidates, 17 patients underwent a second resection, with histopathology confirming tumor in 14 and showing predom- 
inantly radiation necrosis in 3 . Given the expected bias that would be introduced in the calculation of PFS and OS by including patients with pathologically proved radiation necrosis, these 3 patients were excluded. An additional 2 patients who were excluded had a gross total resection with marked FLAIR hyperintensity within the surgical cavity (presumed to be blood products or proteinaceous material) with associated high RSI and low ADC signal, which essentially masked any usable diffusion signal at the margins of the surgical cavity. Tumor progression was determined on the basis of consensus between the treating neuro-oncologist and neuroradiologist by using the Response Assessment in Neuro-Oncology criteria. ${ }^{21}$ In case of no progression or death, PFS was censored at the date of last stable imaging and OS was censored at the date of last contact.

\section{MR Imaging Acquisition and Image Preprocessing}

MR imaging was performed on a 3T Signa Excite HDx scanner (GE Healthcare, Milwaukee, Wisconsin) equipped with an 8-channel head coil. The imaging protocol included pre- and postgadolinium $3 \mathrm{D}$ volumetric T1-weighted inversion recovery spoiled gradient-recalled sequences $(\mathrm{TE} / \mathrm{TR}=2.8 / 6.5 \mathrm{~ms}$, $\mathrm{TI}=$ $450 \mathrm{~ms}$, flip angle $=8^{\circ}, \mathrm{FOV}=24 \mathrm{~cm}$, matrix $=0.93 \times 0.93 \times 1.2$ $\mathrm{mm})$ and a $3 \mathrm{D}$ T2-weighted FLAIR sequence $(\mathrm{TE} / \mathrm{TR}=126 / 6000$ $\mathrm{ms}, \mathrm{TI}=1863 \mathrm{~ms}, \mathrm{FOV}=24 \mathrm{~cm}$, matrix $=0.93 \times 0.93 \times 1.2$ $\mathrm{mm})$. For RSI, a single-shot pulsed-field gradient spin-echo EPI sequence was used $(\mathrm{TE} / \mathrm{TR}=96 \mathrm{~ms} / 17$ seconds, $\mathrm{FOV}=24 \mathrm{~cm}$, matrix $=96 \times 96 \times 48$, voxel size $=2.5 \mathrm{~mm}$ ) with 4 b-values $\left(b=0,500,1500\right.$, and $\left.4000 \mathrm{~s} / \mathrm{mm}^{2}\right)$, with 6,6 , and 15 unique diffusion directions for each nonzero b-value, respectively ( $\sim 8 \mathrm{~min}$ utes scan time).

Before analysis, raw data were corrected for geometric distortions due to susceptibility, gradient nonlinearities, and eddy currents. $^{22}$ This correction was followed by correction of patient motion and rigid registration of the pre- and postcontrast $3 \mathrm{D}$ inversion recovery spoiled gradient-recalled images and the FLAIR images to each other by using in-house software. The diffusion maps were registered to the postcontrast images through the $\mathrm{B} 0$ images ( $b=0 \mathrm{~mm}^{2} / \mathrm{s}$ volume), which were registered to the FLAIR images.

ADC values were calculated from a tensor fit to the $b=0,500$, and $1500 \mathrm{~s} / \mathrm{mm}^{2}$ data. Technical details of the RSI mathematic framework are described in their entirety elsewhere, ${ }^{15-17}$ and the model used has been applied in other recent publications. ${ }^{20}$ Briefly, the measured signal in each voxel was modeled as the sum of signals from 4 distinct tissue compartments: (1) the signal from water trapped within small spheric cells that is restricted in all directions, (2) the signal from water trapped in elongated neuronal processes (ie, neurites) that is restricted in the transverse direction, (3) the signal from extracellular water that is hindered by cells and neuronal processes, and (4) the signal from free water residing in CSF-filled compartments. RSI “cellularity" estimates were computed by combining the signal fraction from the intracellular compartment (1) with the isotropic restricted component of the neurite compartment (2). The RSI cellularity maps were finally transformed to a standard $z$ score by scaling each patient's data by the population mean and SD in normal-appearing white matter of all patients in this study.

\section{VOIs}

Contrast-enhanced volumes $\left(\mathrm{CE}_{\mathrm{vol}}\right)$ and FLAIR hyperintensity (FLAIR-HI) volumes $\left(\right.$ FLAIR $\left._{\mathrm{vol}}\right)$, excluding the resection cavity and intrinsically $\mathrm{T} 1$ hyperintense regions (ie, postsurgical blood products), were segmented semiautomatically (Amira software package; Visage Imaging, San Diego, California.) on the coregistered postcontrast $3 \mathrm{D}$ inversion recovery spoiled gradient-recalled images and FLAIR images by a single expert image analyst with 8 years of experience. For patients whose pre-RT scan was acquired within 2 weeks after the operation, the immediate postsurgery scan was used to exclude areas of restricted diffusion caused by resection-induced cytotoxic edema.

\section{Imaging Metrics}

In this study, we chose to evaluate the utility of both the HC intensities and $\mathrm{HC}$ volume fractions as prognostic factors of PFS and OS. On the basis of a recent study, ${ }^{19}$ the 90 th percentile of RSI cellularity values was selected for our analysis and the HC volume fraction was defined as the volume having an RSI cellularity $z$ score of $\geq 1.5$ within the CE and FLAIR-HI VOIs because the HC volume estimated with this pre-RT normalized ADC threshold was successful in stratifying survival. ${ }^{9}$ Imaging metrics included in the analysis were the following: FLAIR volume $\left(\right.$ FLAIR $\left._{\mathrm{vol}}\right), \mathrm{CE}$ volume $\left(\mathrm{CE}_{\mathrm{vol}}\right)$, the 90th percentile of RSI cellularity values in the CE (RSI-CE ${ }_{90 \%}$ ) and in the FLAIR-HI (RSI-FLAIR ${ }_{90 \%}$ ) VOIs and the RSI-based HC volume fraction in the CE (RSI-CE $E_{\mathrm{vf}}$ ) and the FLAIR-HI (RSI-FLAIR ${ }_{\mathrm{vf}}$ ) VOIs. The following ADC metrics were estimated for comparing against the respective RSI metrics: the 10th percentile of $\mathrm{ADC}^{11}$ in the $\mathrm{CE}$ (ADC-CE $\mathrm{C}_{10 \%}$ ) and in the FLAIR-HI (ADC-FLAIR ${ }_{10 \%}$ ) VOIs and the ADC-based HC volume fraction (ADC $z$ score of $\leq 1.5$ ) in the CE $\left(A D C-\mathrm{CE}_{\mathrm{vf}}\right)$ and FLAIR-HI (ADC-FLAIR $\left.{ }_{\mathrm{vf}}\right)$ VOIs.

\section{Statistical Analysis}

Univariate Cox proportional hazards $(\mathrm{CPH})$ models were used to determine the contribution of resection type (subtotal resection, gross total resection), age, and sex to PFS and OS. Multivariate $\mathrm{CPH}$ models that included a single imaging metric combined with any significant clinical covariates (namely age, gender and resection type) were used to determine the relationship between each continuous pre-RT imaging metric and PFS/OS. Kaplan-Meier curves for the imaging metrics that were significant in the multivariate $\mathrm{CPH}$ models were obtained by dichotomizing the population on the basis of their median value and were compared by using a log-rank test. Due to the exploratory nature of the study, we did not control for type I error. $P$ values $<.05$ were considered statistically significant. Statistical analysis was performed by using R Version 3.2.2 statistical and computing software (http://www.r-project.org/). ${ }^{23}$

\section{RESULTS}

\section{Clinical}

Median PFS in the final cohort $(n=40)$ was 8.4 months, and median OS was 19.5 months. Nine patients progressed within 6 months, 24 patients progressed within 12 months, 30 patients 
progressed within 18 months, and 33 patients progressed within 24 months. The results of univariate $\mathrm{CPH}$ analyses for the clinical and imaging metrics are summarized in Table 2. CPH models revealed that the resection type (subtotal resection-1 versus gross total resection-2) had a trend toward significance for PFS and was significant for OS (Table 2); hence, it was included as a covariate in multivariate $\mathrm{CPH}$ analyses. Age and sex (male-1, female-2) were not predictive of PFS or OS in this cohort. Boxplots of the imaging metrics are shown in Fig 1, and the boxplots split by the median PFS and OS excluding the values from the censored patients for the relevant metrics that achieved significance in Table 3 are shown in On-line Fig 1.
Table 2: Results of univariate CPH analyses of the continuous clinical imaging metrics

\begin{tabular}{|c|c|c|c|c|}
\hline \multirow[b]{2}{*}{ Metrics } & \multicolumn{2}{|r|}{ PFS } & \multicolumn{2}{|r|}{ OS } \\
\hline & $P$ Value & $\mathrm{HR}(95 \% \mathrm{Cl})$ & $P$ Value & $\mathrm{HR}(95 \% \mathrm{Cl})$ \\
\hline Age & .562 & $0.992(0.965-1.019)$ & .857 & $0.997(0.965-1.03)$ \\
\hline Sex & .063 & $0.512(0.253-1.037)$ & .376 & $0.699(0.316-1.546)$ \\
\hline Resection type & .066 & $0.511(0.249-1.046)$ & $.002^{\mathrm{a}}$ & $0.235(0.095-0.58)$ \\
\hline FLAIR $_{\text {vol }}$ & .381 & 1.004 (0.994-1.014) & .404 & $1.005(0.994-1.016)$ \\
\hline $\mathrm{CE}_{\mathrm{vol}}$ & $.047^{\mathrm{a}}$ & 1.04 (1.001-1.08) & $.010^{\mathrm{a}}$ & $1.06(1.014-1.108)$ \\
\hline $\mathrm{ADC} \mathrm{FLAIR}_{\mathrm{vf}}$ & .397 & 0.939 (0.811-1.087) & .52 & $0.936(0.766-1.145)$ \\
\hline$A D C-C E_{v f}$ & .804 & $1.011(0.925-1.105)$ & .932 & 0.995 (0.891-1.112) \\
\hline ADC-FLAIR $10 \%$ & .384 & $1.001(0.999-1.004)$ & .624 & $1.001(0.998-1.004)$ \\
\hline$A D C-C E_{10 \%}$ & .735 & 0.999 (0.997-1.002) & .704 & $1.001(0.998-1.003)$ \\
\hline RSI-FLAIR ${ }_{v f}$ & $.038^{\mathrm{a}}$ & 1.031 (1.002-1.061) & $.006^{\mathrm{a}}$ & $1.051(1.015-1.089)$ \\
\hline $\mathrm{RSI}-\mathrm{CE}_{\mathrm{vf}}$ & .12 & $1.03(0.992-1.069)$ & $.021^{\mathrm{a}}$ & 1.048 (1.007-1.091) \\
\hline RSI-FLAIR $_{90 \%}$ & .092 & $1.522(0.934-2.482)$ & $.028^{\mathrm{a}}$ & $2.041(1.081-3.855)$ \\
\hline $\mathrm{RSI}-\mathrm{CE}_{90 \%}$ & .154 & $1.507(0.858-2.649)$ & .099 & $1.822(0.893-3.716)$ \\
\hline
\end{tabular}

a Significant.
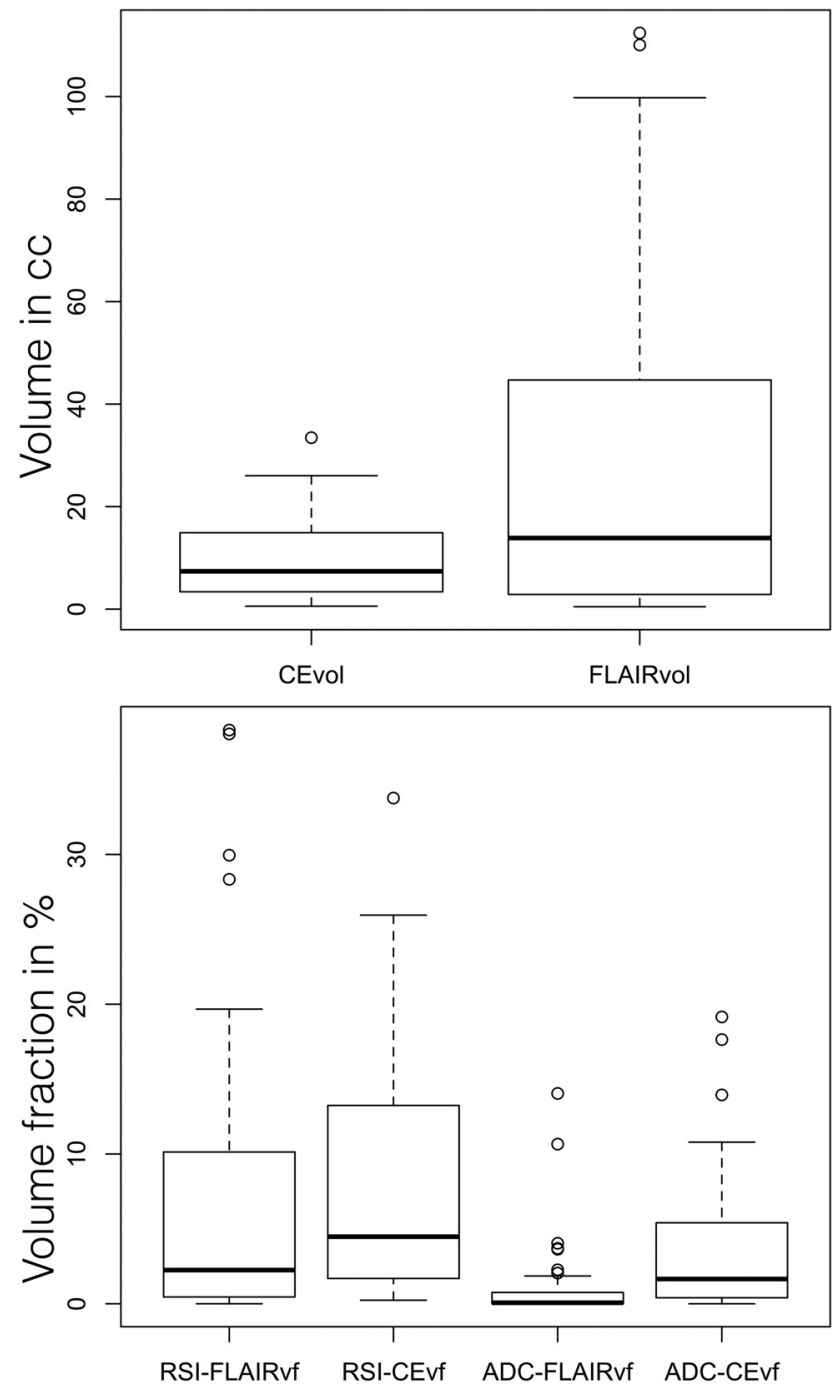

FIG 1. Boxplots of the imaging metrics.

\section{CE and FLAIR-HI Volumes}

In a univariate $\mathrm{CPH}$ analysis, $\mathrm{CE}_{\mathrm{vol}}$ was significantly associated with PFS and OS (Table 2). When adjusted for resection type, $\mathrm{CE}_{\mathrm{vol}}$ was no longer significant for PFS and had a trend toward significance for OS $(P=.063$, Table 3$)$. The Kaplan-Meier curves of the 2 groups obtained by a median split and compared by using the logrank test were significantly different for $\operatorname{PFS}\left(\chi^{2}[1]=4.7, P=.029\right)$ but not for OS $\left(\chi^{2}[1]=3.1, P=.078\right.$; Fig 2$)$. Based on this stratification, the median PFS for the 2 groups was 218 and 338.5 days. FLAIR was not associated with PFS or OS.
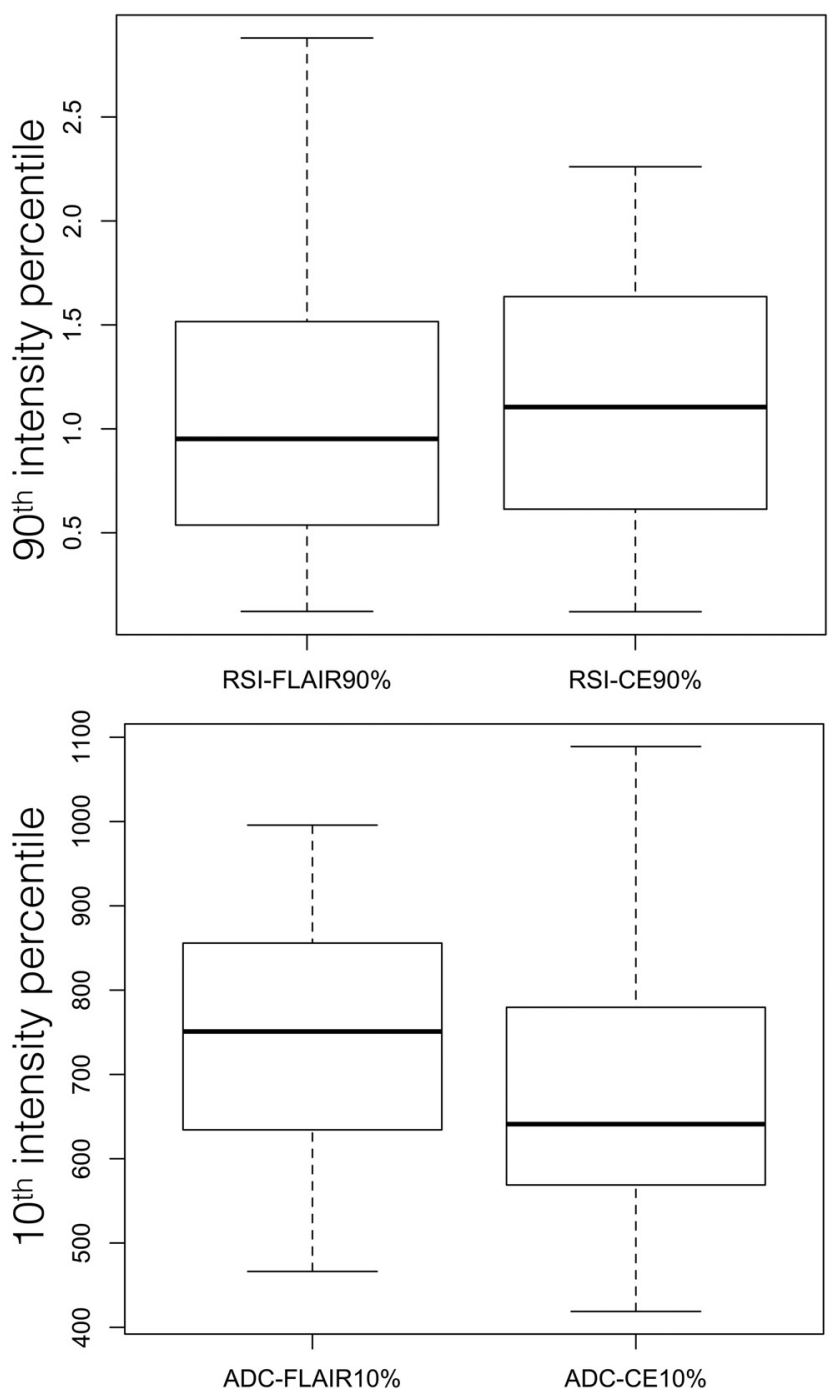


\section{RSI and ADC Metrics}

In a multivariate $\mathrm{CPH}$ analysis, RSI-FLAIR ${ }_{90 \%}$ was significantly associated with OS $(P=.043)$ so that higher RSI intensities were associated with shorter survival (Table 3 ) and RSIFLAIR $_{\mathrm{vf}}$ was significantly associated with both PFS $(P=.036)$ and $\mathrm{OS}(P=.007$, Table 3$)$ so that higher HC volume fractions were associated with earlier progression and shorter survival.

Table 3: Results of multivariate CPH analyses of the continuous imaging metrics covaried with resection type

\begin{tabular}{|c|c|c|c|c|}
\hline \multirow[b]{2}{*}{ Metrics } & \multicolumn{2}{|r|}{ PFS } & \multicolumn{2}{|r|}{ OS } \\
\hline & $P$ Value & HR $(95 \% \mathrm{Cl})$ & $P$ Value & $\mathrm{HR}(95 \% \mathrm{Cl})$ \\
\hline $\mathrm{RSI} \mathrm{FLAIR}_{\mathrm{vf}}$ & $.036^{\mathrm{a}}$ & $1.033(1.002-1.064)$ & $.007^{\mathrm{a}}$ & $1.057(1.015-1.100)$ \\
\hline $\mathrm{RSI}-\mathrm{CE}_{\mathrm{vf}}$ & .258 & $1.022(0.984-1.062)$ & .183 & $1.028(0.987-1.070)$ \\
\hline RSI-FLAIR $_{90 \%}$ & .097 & $1.535(0.925-2.545)$ & $.043^{\mathrm{a}}$ & $2.111(1.024-4.350)$ \\
\hline $\mathrm{RSI}-\mathrm{CE}_{90 \%}$ & .210 & $1.439(0.814-1.543)$ & .217 & $1.568(0.767-3.203)$ \\
\hline FLAIR $_{\text {vol }}$ & .845 & 1.001 (0.990-1.012) & .979 & 1.000 (0.989-1.011) \\
\hline $\mathrm{CE}_{\mathrm{vol}}$ & .165 & 1.029 (0.988-1.073) & $.063^{\mathrm{a}}$ & 1.044 (0.998-1.092) \\
\hline
\end{tabular}

a Significant.
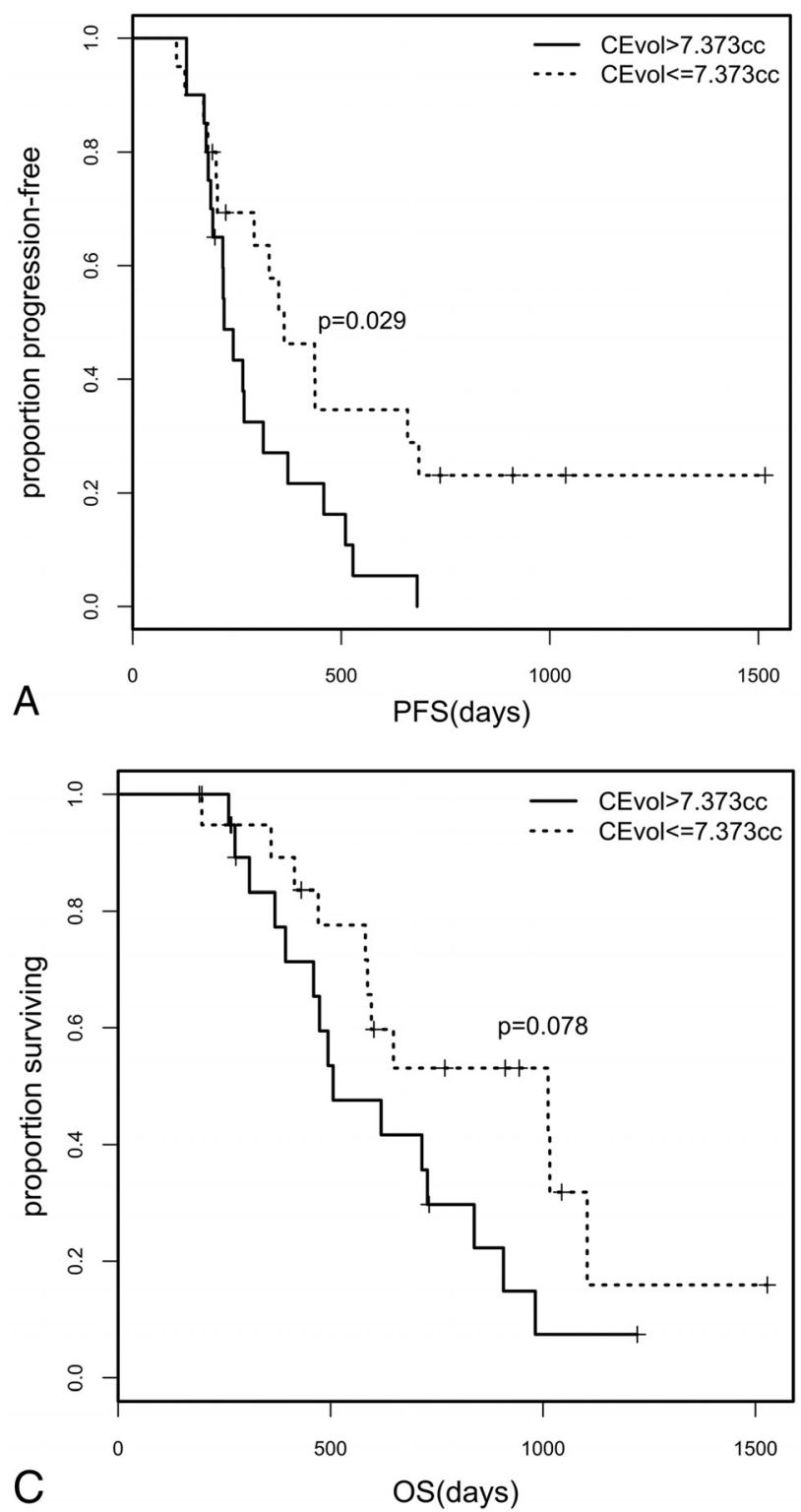

None of the RSI metrics in the CE VOI were significant for PFS/OS.

The Kaplan-Meier curves of the 2 groups obtained by a median split of RSI-FLAIR ${ }_{\mathrm{vf}}$ were significantly different for both PFS $\left(\chi^{2}[1]=6.1, P=.013\right)$ and $\mathrm{OS}\left(\chi^{2}[1]=7.7, P=.005\right.$; Fig 2$)$. The median PFS of the 2 groups was 201.5 and 367.5 days, and the median OS of the 2 groups was 451 and 750.5 days. Despite a strong trend, stratification by the median RSI-FLAIR ${ }_{90 \%}$ did not yield significant group differences in PFS $\left(\chi^{2}[1]=\right.$ $2.8, P=.095)$ and $\mathrm{OS}\left(\chi^{2}[1]=3.4, P=\right.$ $.065)$.

To understand the influence of the threshold for defining HC volume fraction on the prognostic value, we repeated the analysis with $\mathrm{HC}$ volume fraction defined with a threshold of RSI

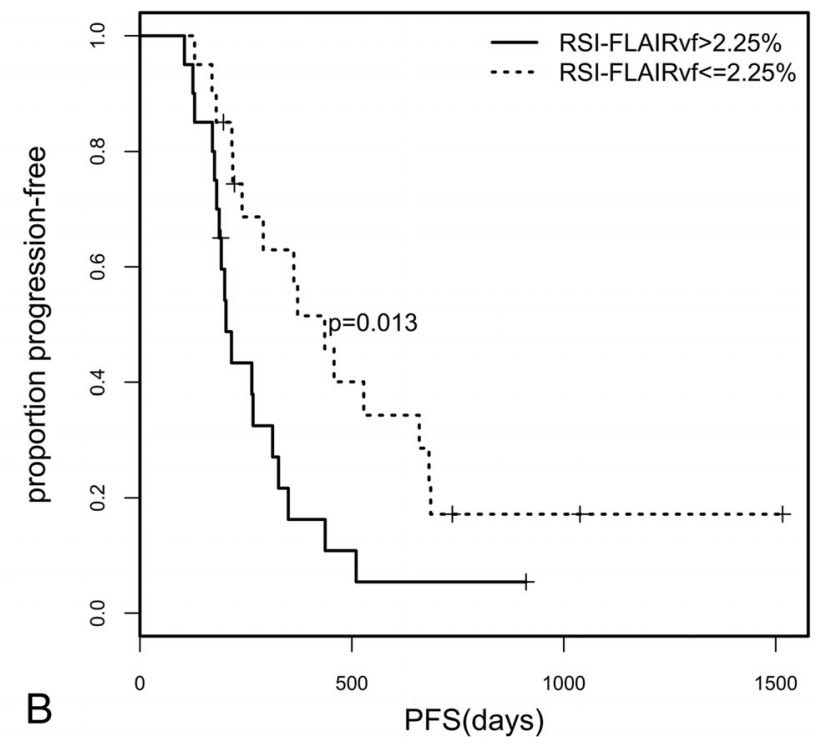


$(B)$ and $O S(D)$.

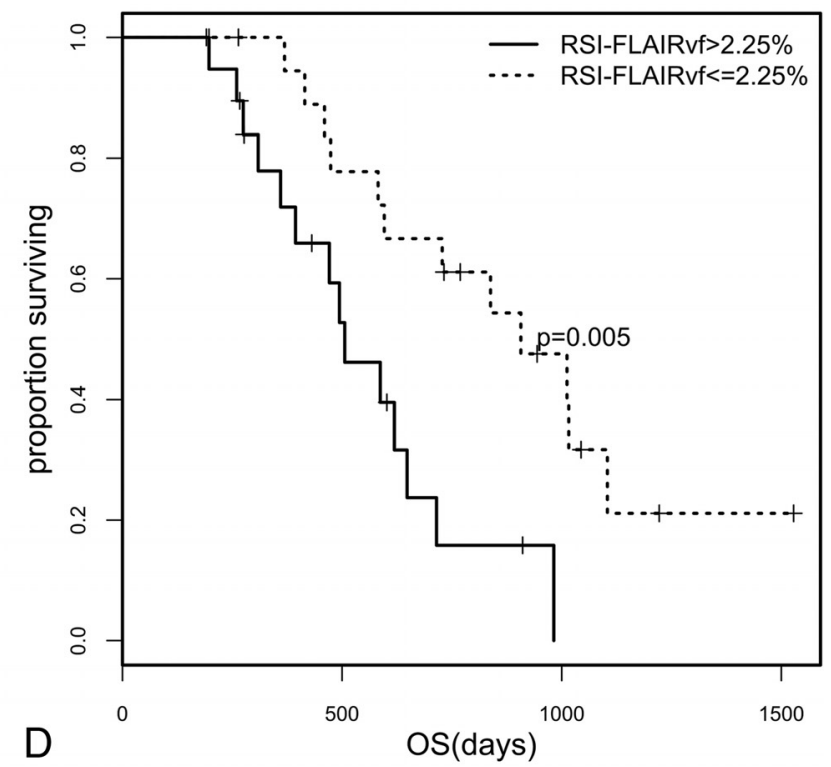






FIG 3. Shown here are the axial T1-postcontrast, FLAIR, ADC, and RSI-cellularity $z$ score maps acquired postsurgery but pre-RT for 2 patients, $A$ and $B$. The VOI contours are shown in red and green for the CE and FLAIR-HI, respectively. Patient A is a 63-year-old man with a right posterior frontal GBM who underwent subtotal resection. This patient had high RSI cellularity in the FLAIR-HI region. Although there is corresponding ADC hypointensity in this region, it is subtle and inconspicuous. He had a shorter PFS and OS (PFS, 4.2 months; OS, 6.6 months) than patient B. Patient $B$ is a 31-year-old woman with a right frontal GBM. There are no areas of high RSI cellularity or low ADC signal in the FLAIR-HI or CE region. She had a correspondingly longer PFS (PFS, 14.5 months; OS, 19.9 months).

cellularity $z$ score $\geq 1$ and $\geq 2$. RSI-FLAIR vf $_{\text {at }} z \geq 1$ was significantly associated with PFS $(P=.03$, hazard ratio $[\mathrm{HR}]=1.023)$ and $\mathrm{OS}(P=.042, \mathrm{HR}=1.027)$. While RSI-FLAIR ${ }_{\mathrm{vf}}$ at $\mathrm{z} \geq 2$ was significant for OS $(P=.009, \mathrm{HR}=1.096)$, it was not significant for PFS $(P=.09, \mathrm{HR}=1.043)$. Similarly, we also explored the prognostic value of the absolute $\mathrm{HC}$ volume with $z \geq 1.5$, which were prognostic for $\mathrm{OS}\left(\mathrm{HC}_{\mathrm{vol}}\right.$ in $\mathrm{CE}: P=.018, \mathrm{HR}=1.543$; FLAIR-HI: $P=.042, \mathrm{HR}=1.262$ ) in a multivariate $\mathrm{CPH}$ analysis. $\mathrm{HC}_{\mathrm{vol}}$ within $\mathrm{CE}$ was significant for PFS in a univariate analysis $(P=.025, \mathrm{HR}=1.141)$ but was not significant in a multivariate analysis with resection type as a covariate.

In a univariate $\mathrm{CPH}$ analysis, none of the $\mathrm{ADC}$ metrics were associated with PFS (Table 2). The absolute ADC HC volumes with $z \leq 1.5$ within the FLAIR-HI VOI were significantly prognostic of $\mathrm{OS}$ in a univariate $\mathrm{CPH}$ analysis $(P=.015, \mathrm{HR}=58.89)$ but only had a trend toward significance $(P=.079, \mathrm{HR}=20.06)$ after accounting for resection type in a multivariate $\mathrm{CPH}$ analysis and were not associated with PFS (univariate: $P=.138, \mathrm{HR}=$ 4.835). The ADC HC volumes within the CE VOI were not associated with outcomes.

Representative images of 2 patients in this cohort with short (patient A) and long (patient B) PFS are shown in Fig 3. Patient A had lower $\mathrm{CE}_{\mathrm{vol}}$, FLAIR ${ }_{\mathrm{vol}}$, and ADC-FLAIR ${ }_{10 \%}$ but higher RSIFLAIR $_{90 \%}$, RSI-FLAIR ${ }_{v f}$, and ADC-FLAIR ${ }_{v f}$ than patient B, likely reflecting higher tumor cellularity in the patient's FLAIR-HI region. RSI-cellularity maps exhibited greater conspicuity in this region compared with ADC maps. Accordingly, patient A had shorter PFS and OS compared with patient B.

\section{DISCUSSION}

In the management of a highly aggressive tumor like GBM, the ability to stratify patient survival postoperatively is important be- cause this information can directly impact therapeutic decision-making. In this study, we found RSI metrics to better stratify patients according to both PFS and OS compared with conventional imaging and ADC metrics. Unfortunately, $\mathrm{CE}_{\mathrm{vol}}$ and $\mathrm{FLAIR}_{\mathrm{vol}}$ may have limited prognostic value in this setting once the extent of the resection is considered. Patients with glioblastoma often have a more infiltrative tumor pattern and possibly a more hypoxic tumor biology, ${ }^{24}$ with some of them responding poorly to radiation therapy or conventional chemotherapy. ${ }^{25}$ The nonspecificity of these conventional metrics could potentially be due to the hypoxic tumors not having contrast enhancement and the presence of infiltrative tumors not having a noticeable signal in both T1 and T2 images. Hence, there is a need for better imaging metrics that perform reliably and may aid in identification of patients with GBM at high risk for early recurrence and worse survival.

Among the imaging metrics, we found that the pre-RT RSI-derived measures of cellularity within the FLAIR-HI region were associated with PFS and OS. The prognostic value of RSI appeared robust to the threshold used to determine the $\mathrm{HC}$ volume. Conversely, ADC metrics within the FLAIR-HI region were not associated with outcomes. Tumor progression results in areas of increased tumor cellularity (ie, decrease in diffusivity) and also areas of increased edema (ie, increase in diffusivity), both of which can occur simultaneously within an imaging voxel. Furthermore, postsurgery but pre-RT, a significant portion of the enhancing $\mathrm{HC}$ volume of the tumor has probably been resected and the residual $\mathrm{HC}$ tumor is interspersed with edema. Because ADC is a composite measure, the effects of these 2 opposing factors may cancel each other out, therefore limiting the prognostic value of ADC. RSI overcomes this limitation by separating the diffusivities associated with intracellular, restricted diffusion from the extracellular effects of edema. In contrast to a previous study, ${ }^{9}$ the absolute ADC HC volume was not significantly associated with OS, and this finding might be due to the inclusion of resection type as a covariate in the analysis. Even though the absolute RSI HC volume was prognostic of OS, the volume fraction was a stronger prognostic metric of PFS/OS. We did not find an association between any of our diffusion metrics in the CE region, similar to findings of previous studies. ${ }^{9,10}$ Although the reason for this outcome is not clear, it is likely that the $\mathrm{CE}$ region was quite limited in size in most patients, given the short interval between surgery and RT.

Other advanced imaging techniques, including perfusion, ${ }^{10}$ MR spectroscopic imaging (MRSI), ${ }^{26}$ and $\mathrm{PET}^{27}$ have also been shown to stratify outcomes in patients with GBM. While perfusion imaging provides information on vascular density and flow, MRSI and PET provide metabolic information. Pre-RT perfusion 
imaging metrics have been shown to predict PFS, ${ }^{10}$ but perfusion is limited in the central core of a solid tumor with hypoxic cancer cells ${ }^{28}$ and with antiangiogenic therapy in which the vasculature is normalized, removing the leaky vessels in the tumor. ${ }^{29}$ Pre-RT MRSI metrics are associated with both PFS and OS $^{26}$ but have lower spatial resolution compared with conventional, perfusion, and diffusion MR imaging. Although diffusion metrics should perform better than perfusion and MRSI with hypoxic tumor and antiangiogenic therapy, none of the previous studies have shown an association of pre-RT diffusion with outcomes. Here, we show that RSI-based cellularity is a prognostic metric of PFS and OS and may offer advantages over perfusion and MRSI in the pre-RT setting.

One of the main limitations of the current study is the small sample size obtained at a single institution (University of California, San Diego). The current study represents our effort to explore the clinical utility of RSI in a highly controlled study in which all the patients were scanned in the same scanner and all images were processed in a highly uniform manner, which included robust corrections for motion and geometric distortions. Given our modest size, we might be underpowered to detect smaller associations between some of our imaging metrics and survival. The generalizability and reproducibility of our results will need to be tested in prospective, multisite clinical trials across multiple vendor platforms. A second limitation is that the heterogeneity of the therapeutic approaches that each patient received following standard RT and temozolomide made it difficult to stratify patients according to treatment regimen. However, our major finding is that RSI performed better than ADC and conventional imaging in the same patient cohort, which is not confounded by betweenpatient treatment variance. Future studies with cohorts large enough to stratify patients according to the additional therapies received would be of great benefit for better delineating predictors of response to various therapies. Another possible limitation is that the tumors were segmented by a single imaging expert. Thus, intra- and interobserver variability of the tumor segmentations was not evaluated. In addition, although all patients had pathology-confirmed GBMs at the outset, histologic validation of tumor progression was not available for all patients. Furthermore, correlation with genomic information and molecular markers (such as MGMT and IDH status), which are known to provide prognostic information, could not be performed because these data were only available for a subset of the cohort (Table 1). Molecular information is now systematically collected for patients with GBM at our institution, facilitating future studies investigating the prognostic value of these markers. Although the results from our recent preclinical study ${ }^{30}$ show that the RSI cellularity metric correlates with histopathologic markers of cellularity, additional validation in patients with GBM is warranted.

\section{CONCLUSIONS}

Following the operation but before initiating RT, RSI-derived cellularity in the FLAIR-HI region performed better than ADC and conventional imaging for risk stratification in patients with GBM. Therefore, RSI could be potentially useful for identifying patients at highest risk for early progression and shorter survival. How- ever, future studies with larger sample sizes are needed to explore its predictive ability.

\section{ACKNOWLEDGMENTS}

We would like to thank patients at the University of California, San Diego, Moores Cancer Center Neuro-Oncology Program, for their generous participation.

Disclosures: Anitha Priya Krishnan—RELATED: Grant: National Science Foundation and National Institutes of Health, Comments: I was funded for my postdoctoral work from a National Science Foundation grant (Principal Investigator: Nathan S. White) and a National Institutes of Health grant (Principal Investigator: Anders M. Dale). Tyler M. Seibert-UNRELATED: Grants/Grants Pending: Varian Medical Systems, Comments: research grant*; Payment for Development of Educational Presentations: WebMD, Comments: honoraria for providing continuing education materials for physicians. Anders M. Dale-RELATED: Grant: GE Healthcare, Comments: I am the principal investigator of a comprehensive research agreement between GE Healthcare and the University of California, San Diego*; Other: Comments: I am a coinventor on a patent covering the restriction spectrum imaging technology used in the article. The patent is assigned to the University of California*; UNRELATED: Board Membership: Human Longevity and CorTechs Labs, Comments: I serve on the scientific advisory boards of Human Longevity and CorTechs Labs; Grants/Grants Pending: National Institutes of Health, Comments: numerous research grants*; Patents (Planned, Pending, or Issued): Comments: numerous patents*; Stock/Stock Options: CorTechs Labs, Comments: I am a founder of and hold equity in CorTechs Labs. Jona A. Hattangadi-Gluth—UNRELATED: Grants/Grants Pending: Varian Medical Systems, Comments: A research grant from Varian Medical Systems was made to my institution to fund pilot imaging projects. One of these is my project as Principal Investigator*. Carrie McDonald-RELATED: Grant: American Cancer Society*. *Money paid to the institution.

\section{REFERENCES}

1. Dolecek TA, Propp JM, Stroup NE, et al. CBTRUS statistical report: primary brain and central nervous system tumors diagnosed in the United States in 2005-2009. Neuro Oncol 2012;14(suppl 5):v1-49.21 CrossRef Medline

2. Stupp R, Mason WP, van den Bent MJ, et al; European Organisation for Research and Treatment of Cancer Brain Tumor and Radiotherapy Groups, National Cancer Institute of Canada Clinical Trials. Radiotherapy plus concomitant and adjuvant temozolomide for glioblastoma. N Engl J Med 2005;352:987-96 CrossRef Medline

3. Carlsson SK, Brothers SP, Wahlestedt C. Emerging treatment strategies for glioblastoma multiforme. EMBO Mol Med 2014;6:1359-70 CrossRef Medline

4. Tsien CI, Brown D, Normolle D, et al. Concurrent temozolomide and dose-escalated intensity-modulated radiation therapy in newly diagnosed glioblastoma. Clin Cancer Res 2012;18:273-79 CrossRef Medline

5. Pope WB, Lai A, Mehta R, et al. Apparent diffusion coefficient histogram analysis stratifies progression-free survival in newly diagnosed bevacizumab-treated glioblastoma. AJNR Am J Neuroradiol 2011;32:828-31 CrossRef Medline

6. Ellingson BM, Sahebjam S, Kim HJ, et al. Pretreatment ADC histogram analysis is a predictive imaging biomarker for bevacizumab treatment but not chemotherapy in recurrent glioblastoma. AJNR Am J Neuroradiol 2014;35:673-79 CrossRef Medline

7. Higano S, Yun X, Kumabe T, et al. Malignant astrocytic tumors: clinical importance of apparent diffusion coefficient in prediction of grade and prognosis. Radiology 2006;241:839-46 CrossRef Medline

8. Mulkern RV, Gudbjartsson H, Westin C-F, et al. Multi-component apparent diffusion coefficients in human brain. NMR Biomed 1999; 12:51-62 Medline

9. Saraswathy S, Crawford F, Lamborn K, et al. Evaluation of MR markers that predict survival in patients with newly diagnosed GBM prior to adjuvant therapy. J Neurooncol 2009;91:69-81 CrossRef Medline

10. Li Y, Lupo JM, Polley MY, et al. Serial analysis of imaging parame- 
ters in patients with newly diagnosed glioblastoma multiforme. Neuro Oncol 2011;13:546-57 CrossRef Medline

11. Wen Q, Jalilian L, Lupo JM, et al. Comparison of ADC metrics and their association with outcome for patients with newly diagnosed glioblastoma being treated with radiation therapy, temozolomide, erlotinib and bevacizumab. J Neurooncol 2015;121:331-39 CrossRef Medline

12. Pope WB, Kim HJ, Huo J, et al. Recurrent glioblastoma multiforme: ADC histogram analysis predicts response to bevacizumab treatment. Radiology 2009;252:182-89 CrossRef Medline

13. Crawford FW, Khayal IS, McGue C, et al. Relationship of pre-surgery metabolic and physiological MR imaging parameters to survival for patients with untreated GBM. J Neurooncol 2009;91:337-51 CrossRef Medline

14. Ellingson BM, Cloughesy TF, Zaw T, et al. Functional diffusion maps (fDMs) evaluated before and after radiochemotherapy predict progression-free and overall survival in newly diagnosed glioblastoma. Neuro Oncol 2012;14:333-43 CrossRef Medline

15. White NS, Leergaard TB, D'Arceuil H, et al. Probing tissue microstructure with restriction spectrum imaging: histological and theoretical validation. Hum Brain Mapp 2013;34:327-46 CrossRef Medline

16. White NS, Dale AM. Optimal diffusion MRI acquisition for fiber orientation density estimation: an analytic approach. Hum Brain Mapp 2009;30:3696-703 CrossRef Medline

17. White NS, Dale AM. Distinct effects of nuclear volume fraction and cell diameter on high b-value diffusion MRI contrast in tumors. Magn Reson Med 2014;72:1435-43 CrossRef Medline

18. White NS, McDonald CR, Farid N, et al. Improved conspicuity and delineation of high-grade primary and metastatic brain tumors using "restriction spectrum imaging": quantitative comparison with high B-value DWI and ADC. AJNR Am J Neuroradiol 2013;34:95864, S1 CrossRef Medline

19. McCammack KC, Kane CJ, Parsons JK, et al. In vivo prostate cancer detection and grading using restriction spectrum imaging-MRI. Prostate Cancer Prostatic Dis 2016;19:168-73 CrossRef Medline
20. McDonald CR, Delfanti RL, Krishnan AP, et al. Restriction spectrum imaging predicts response to bevacizumab in patients with highgrade glioma. Neuro Oncol 2016;18:1579-90 Medline

21. Wen PY, Macdonald DR, Reardon DA, et al. Updated response assessment criteria for high-grade gliomas: response assessment in neuro-oncology working group. J Clin Oncol 2010;28:1963-72 CrossRef Medline

22. Holland D, Kuperman JM, Dale AM. Efficient correction of inhomogeneous static magnetic field-induced distortion in echo planar imaging. Neuroimage 2010;50:175-83 CrossRef Medline

23. R Core Team. R: A Language and Environment for Statistical Computing. R Foundation for Statistical Computing. Vienna; the R Foundation for Statistical Computing; 2015

24. Vanan MI, Eisenstat DD. Management of high-grade gliomas in the pediatric patient: past, present, and future. Neurooncol Pract 2014; 1:145-57 CrossRef Medline

25. Haar CP, Hebbar P, Wallace GC 4th, et al. Drug resistance in glioblastoma: a mini review. Neurochem Res 2012;37:1192-200 CrossRef Medline

26. Li Y, Lupo JM, Parvataneni R, et al. Survival analysis in patients with newly diagnosed glioblastoma using pre- and postradiotherapy MR spectroscopic imaging. Neuro Oncol 2013;15:607-17 CrossRef Medline

27. Tralins KS, Douglas JG, Stelzer KJ, et al. Volumetric analysis of $18 \mathrm{~F}-$ FDG PET in glioblastoma multiforme: prognostic information and possible role in definition of target volumes in radiation dose escalation. J Nucl Med 2002;43:1667-73 Medline

28. Eales KL, Hollinshead KER, Tennant DA. Hypoxia and metabolic adaptation of cancer cells. Oncogenesis 2016;5:e190 CrossRef Medline

29. Plate KH, Scholz A, Dumont DJ. Tumor angiogenesis and anti-angiogenic therapy in malignant gliomas revisited. Acta Neuropathol 2012;124:763-75 CrossRef Medline

30. Hope TR, White NS, Kuperman J, et al. Demonstration of nongaussian restricted diffusion in tumor cells using diffusion timedependent diffusion-weighted magnetic resonance imaging contrast. Front Oncol 2016;6:179 CrossRef Medline 